\title{
The Zero-Removing Property in Hilbert Spaces of Entire Functions Arising in Sampling Theory
}

\author{
A. G. García and M. A. Hernández-Medina
}

\begin{abstract}
In the topic of sampling in reproducing kernel Hilbert spaces, sampling in Paley-Wiener spaces is the paradigmatic example. A natural generalization of Paley-Wiener spaces is obtained by substituting the Fourier kernel with an analytic Hilbert-space-valued kernel $K$. Thus we obtain a reproducing kernel Hilbert space $\mathcal{H}_{K}$ of entire functions in which the Kramer property allows to prove a sampling theorem. A necessary and sufficient condition ensuring that this sampling formula can be written as a Lagrange-type interpolation series concerns the stability under removal of a finite number of zeros of the functions belonging to the space $\mathcal{H}_{K}$; this is the so-called zero-removing property. This work is devoted to the study of the zero-removing property in $\mathcal{H}_{K}$ spaces, regardless of the Kramer property, revealing its connections with other mathematical fields.
\end{abstract}

Mathematics Subject Classification. 46E22, 42C15, 94A20.

Keywords. Analytic Kramer kernel, Lagrange-type interpolation series, zero-removing property.

\section{Introduction}

Sampling in reproducing kernel Hilbert spaces is nowadays an interesting mathematical topic (see, for instance, Refs. $[8,19,21]$ ). Besides, it has opened new research lines: sampling in unitarily translation invariant reproducing kernel Hilbert spaces or sampling in reproducing Banach spaces (see, for instance, 
Refs. $[13,18,20,21])$. The present work is intimately related with this subject, and an easy motivation can be found in the Lagrange-type interpolatory character of the Shannon sampling theorem which holds for Paley-Wiener spaces. Namely, the Paley-Wiener space $P W_{\pi}$ of bandlimited functions to $[-\pi, \pi]$, i.e.,

$$
P W_{\pi}:=\left\{f \in L^{2}(\mathbb{R}) \cap C(\mathbb{R}) \mid \operatorname{supp} \hat{f} \subseteq[-\pi, \pi]\right\},
$$

where $\hat{f}$ stands for the Fourier transform of $f$, coincides, via the classical Paley-Wiener theorem [28, p. 85], with the space of entire functions $f$ such that $|f(z)| \leq A \mathrm{e}^{\pi|z|}$ on $\mathbb{C}$ for some positive constant $A$, and $\left.f\right|_{\mathbb{R}} \in L^{2}(\mathbb{R})$. In $P W_{\pi}$ the classical Shannon sampling theorem holds: Any $f \in P W_{\pi}$ can be expanded as

$$
f(z)=\sum_{n=-\infty}^{\infty} f(n) \frac{\sin \pi(z-n)}{\pi(z-n)}, \quad z \in \mathbb{C} .
$$

The series converges absolutely and uniformly on horizontal strips of the complex plane. Moreover, the sampling expansion (1) can be written as the Lagrange-type interpolation series

$$
f(z)=\sum_{n=-\infty}^{\infty} f(n) \frac{P(z)}{(z-n) P^{\prime}(n)}, \quad z \in \mathbb{C},
$$

where $P$ stands for the entire function $P(z)=(\sin \pi z) / \pi$, which has only simple zeros at $\mathbb{Z}$.

Since any function $f \in P W_{\pi}$ can be written as

$$
f(z)=\left\langle\frac{\mathrm{e}^{i z}}{\sqrt{2 \pi}}, F\right\rangle_{L^{2}[-\pi, \pi]}, \quad z \in \mathbb{C},
$$

for some function $F \in L^{2}[-\pi, \pi]$, Shannon sampling theory admits a straightforward generalization by substituting the Fourier kernel

$\mathbb{C} \ni z \longmapsto K(z) \in L^{2}[-\pi, \pi]$ such that $K(z)(w):=\mathrm{e}^{i z w} / \sqrt{2 \pi}, \quad w \in[-\pi, \pi]$,

by another abstract kernel $K$ valued in a Hilbert space $\mathcal{H}$. The analytic Kramer sampling theory accomplishes this generalization. Indeed, let $\mathcal{H}$ be a complex, separable Hilbert space with inner product $\langle\cdot,-\rangle_{\mathcal{H}}$ and suppose $K$ is an $\mathcal{H}$ valued analytic function defined on $\mathbb{C}$. For each $x \in \mathcal{H}$, define the function $f_{x}(z)=\langle K(z), x\rangle_{\mathcal{H}}$ on $\mathbb{C}$, and let $\mathcal{H}_{K}$ denote the collection of all such functions $f_{x}$. Furthermore, each element in $\mathcal{H}_{K}$ is an entire function since $K$ is analytic on $\mathbb{C}$. In this setting, an abstract version of the analytic Kramer theorem [16] is obtained by assuming the Kramer property, that is, the existence of two sequences, $\left\{z_{n}\right\}_{n=1}^{\infty}$ in $\mathbb{C}$ and $\left\{a_{n}\right\}_{n=1}^{\infty}$ in $\mathbb{C} \backslash\{0\}$, and a Riesz basis for $\mathcal{H}$ $\left\{x_{n}\right\}_{n=1}^{\infty}$ such that $K\left(z_{n}\right)=a_{n} x_{n}$ for each $n \in \mathbb{N}$. Namely, for any $f_{x} \in \mathcal{H}_{K}$ we have

$$
f_{x}(z)=\sum_{n=1}^{\infty} f_{x}\left(z_{n}\right) \frac{S_{n}(z)}{a_{n}}, \quad z \in \mathbb{C}
$$


where, for each $n \in \mathbb{N}, S_{n}(z)=\left\langle K(z), y_{n}\right\rangle, z \in \mathbb{C}$, and $\left\{y_{n}\right\}_{n=1}^{\infty}$ stands for the dual Riesz basis of $\left\{x_{n}\right\}_{n=1}^{\infty}$ (see Sect. 2 below for the details).

A challenging problem is to give a necessary and sufficient condition to ensure that the above sampling formula can be written as a Lagrange-type interpolation series, that is

$$
f_{x}(z)=\sum_{n=1}^{\infty} f_{x}\left(z_{n}\right) \frac{P(z)}{\left(z-z_{n}\right) P\left(z_{n}\right)}, \quad z \in \mathbb{C},
$$

where $P$ denotes an entire function having only simple zeros at all points of the sequence $\left\{z_{n}\right\}_{n=1}^{\infty}$. The necessary and sufficient condition ensuring when a Kramer sampling expansion (2) can be written as a Lagrange-type interpolation series (3) was proved in [8] for orthogonal sampling formulas, and in [9] for non-orthogonal Riesz basis sampling formulas. Roughly speaking, the aforesaid necessary and sufficient condition concerns the stability of the functions belonging to the space $\mathcal{H}_{K}$ under removal of a finite number of their zeros; in other words,

$$
f \in \mathcal{H}_{K} \text { and } f(a)=0 \text { implies that } \frac{f(z)}{z-a} \in \mathcal{H}_{K} .
$$

This is an ubiquitous algebraic property in the mathematical literature (see Sect. 2 below) and it will be called the zero-removing property (ZR property in short) throughout the paper. The main aim in this paper is to thoroughly study the ZR property in $\mathcal{H}_{K}$ spaces, regardless of the Kramer property, revealing its relationships with other mathematical fields. For instance, Paley-Wiener spaces are particular cases of de Branges spaces [4] where the ZR property holds, and de Branges spaces are particular cases of $\mathcal{H}_{K}$ spaces as well $[10]$.

Next, we outline the organization of the paper, highlighting its significant contributions. In Sect. 2 we introduce the needed preliminaries on spaces $\mathcal{H}_{K}$ : these spaces are reproducing kernel Hilbert spaces (RKHS in short) of entire functions; we briefly recall the sampling result in $\mathcal{H}_{K}$. In Sect. 3 we study some properties of $\mathcal{H}_{K}$ obtained from the Taylor coefficients of the kernel $K$ at a fixed complex point. In particular, the relationship between $\mathcal{H}_{K}$ and the set $\mathcal{P}(\mathbb{C})$ of complex polynomials. In Section 4 we study the zero-removing property at a fixed point; this property can be reduced to a general moment problem. Thus, the zero-removing property at a fixed point depends on the continuity of a certain associated operator which looks like the classical shift operator. Moreover, we give a sufficient condition for the continuity of this operator. The section is closed by studying the local zero-removing property: If the zero-removing property holds for a fixed point, say 0 , it also holds for any $a \in \mathbb{C}$ with $|a|$ small enough. This study is carried out by using the wellknown Fredholm operator theory. Finally, in Sect. 5 we close the paper with an study of the differentiation operator in an $\mathcal{H}_{K}$ space. 


\section{Preliminaries on $\mathcal{H}_{K}$ Spaces}

Suppose we are given a separable complex Hilbert space and an abstract kernel $K$ which is nothing but an $\mathcal{H}$-valued function on $\mathbb{C}$. For each $z \in \mathbb{C}$, set $f_{x}(z):=\langle K(z), x\rangle_{\mathcal{H}}$ for $z \in \mathbb{C}$, and denote by $\mathcal{H}_{K}$ the collection of all such functions $f_{x}, x \in \mathcal{H}$, and let $\mathcal{T}_{K}$ be the mapping

$$
\mathcal{H} \ni x \stackrel{\mathcal{T}_{K}}{\longmapsto} f_{x} \in \mathcal{H}_{K}
$$

If we define the norm $\|f\|_{\mathcal{H}_{K}}:=\inf \left\{\|x\|_{\mathcal{H}}: f=\mathcal{T}_{K} x\right\}$ in $\mathcal{H}_{K}$ (in the sequel we omit the subscript $x$ in $f_{x}$ ), we obtain a reproducing kernel Hilbert space whose reproducing kernel is given by

$$
k(z, w)=\langle K(z), K(w)\rangle_{\mathcal{H}}, \quad z, w \in \mathbb{C} .
$$

(see [24] for the details). Notice that the mapping $\mathcal{T}_{K}$ is an antilinear mapping from $\mathcal{H}$ onto $\mathcal{H}_{K}$. It is injective if and only if the set $\{K(z)\}_{z \in \mathbb{C}}$ is complete in $\mathcal{H}$. In particular, if there exist sequences $\left\{z_{n}\right\}_{n=1}^{\infty} \subset \mathbb{C},\left\{a_{n}\right\}_{n=1}^{\infty} \in \mathbb{C} \backslash\{0\}$ and a Riesz basis $\left\{x_{n}\right\}_{n=1}^{\infty}$ for $\mathcal{H}$ such that $K\left(z_{n}\right)=a_{n} x_{n}$ for any $n \in \mathbb{N}$, then the mapping $\mathcal{T}_{K}$ is an anti-linear isometry from $\mathcal{H}$ onto $\mathcal{H}_{K}$. Recall that a Riesz basis in a separable Hilbert space $\mathcal{H}$ is the image of an orthonormal basis by means of a boundedly invertible operator. Any Riesz basis $\left\{x_{n}\right\}_{n=1}^{\infty}$ has a unique biorthonormal (dual) Riesz basis $\left\{y_{n}\right\}_{n=1}^{\infty}$, i.e., $\left\langle x_{n}, y_{m}\right\rangle_{\mathcal{H}}=\delta_{n, m}$, such that the expansions

$$
x=\sum_{n=1}^{\infty}\left\langle x, y_{n}\right\rangle_{\mathcal{H}} x_{n}=\sum_{n=1}^{\infty}\left\langle x, x_{n}\right\rangle_{\mathcal{H}} y_{n}
$$

hold for every $x \in \mathcal{H}$ (see $[6,28]$ for more details and proofs).

The convergence in the norm $\|\cdot\|_{\mathcal{H}_{K}}$ implies pointwise convergence which is uniform on those subsets of $\mathbb{C}$ where the function $z \mapsto\|K(z)\|_{\mathcal{H}}$ is bounded; in particular, in compact subsets of $\mathbb{C}$ whenever $K$ is a continuous kernel.

Like in the classical case the following result holds: The space $\mathcal{H}_{K}$ is a RKHS of entire functions if and only if the kernel $K$ is analytic in $\mathbb{C}$ ([26, p. 266]). Another characterization of the analyticity of the functions in $\mathcal{H}_{K}$ is given in terms of Riesz bases. Suppose that a Riesz basis $\left\{x_{n}\right\}_{n=1}^{\infty}$ for $\mathcal{H}$ is given and let $\left\{y_{n}\right\}_{n=1}^{\infty}$ be its dual Riesz basis; expanding $K(z)$, where $z \in \mathbb{C}$ is fixed, with respect to the basis $\left\{x_{n}\right\}_{n=1}^{\infty}$ we obtain

$$
K(z)=\sum_{n=1}^{\infty}\left\langle K(z), y_{n}\right\rangle_{\mathcal{H}} x_{n}=\sum_{n=1}^{\infty} S_{n}(z) x_{n} \quad \text { in } \mathcal{H},
$$

where the coefficients

$$
S_{n}(z):=\left\langle K(z), y_{n}\right\rangle_{\mathcal{H}}, \quad z \in \mathbb{C},
$$

as functions in $z \in \mathbb{C}$, are in $\mathcal{H}_{K}$. The following result holds [10]: Let $\left\{x_{n}\right\}_{n=1}^{\infty}$ and $\left\{y_{n}\right\}_{n=1}^{\infty}$ be a pair of dual Riesz bases for $\mathcal{H}$. Then, $\mathcal{H}_{K}$ is RKHS of entire functions if and only if all the functions $S_{n}, n \in \mathbb{N}$, are entire and the function $z \mapsto\|K(z)\|_{\mathcal{H}}$ is bounded on compact sets of $\mathbb{C}$. 


\subsection{Sampling and the Zero-Removing Property in $\mathcal{H}_{K}$ Spaces}

Consider the data

$$
\left\{z_{n}\right\}_{n=1}^{\infty} \in \mathbb{C} \text { and }\left\{a_{n}\right\}_{n=1}^{\infty} \in \mathbb{C} \backslash\{0\} .
$$

Definition 1. An analytic kernel $K: \mathbb{C} \longrightarrow \mathcal{H}$ is said to be an analytic Kramer kernel (with respect to the data (6)) if it satisfies $K\left(z_{n}\right)=a_{n} x_{n}, n \in \mathbb{N}$, for some Riesz basis $\left\{x_{n}\right\}_{n=1}^{\infty}$ of $\mathcal{H}$. A sequence $\left\{S_{n}\right\}_{n=1}^{\infty}$ of functions in $\mathcal{H}_{K}$ is said to have the interpolation property (with respect to the data (6)) if

$$
S_{n}\left(z_{m}\right)=a_{n} \delta_{n, m} .
$$

An analytic kernel $K$ is an analytic Kramer one if and only if the sequence of functions $\left\{S_{n}\right\}_{n=1}^{\infty}$ in $\mathcal{H}_{K}$ given by (5), where $\left\{y_{n}\right\}_{n=1}^{\infty}$ is the dual Riesz basis of $\left\{x_{n}\right\}_{n=1}^{\infty}$, has the interpolation property with respect to the same data $(6)$.

Under the notation introduced so far an abstract version of the classical Kramer sampling theorem [16] holds: First notice that $\lim _{m \rightarrow \infty}\left|z_{m}\right|=+\infty$; otherwise we obtain that any entire function $S_{n}$ is identically zero in $\mathbb{C}$. The anti-linear mapping $\mathcal{T}_{K}$ is a bijective isometry between $\mathcal{H}$ and $\mathcal{H}_{K}$. As a consequence, the functions $\left\{S_{n}=\mathcal{T}_{K}\left(y_{n}\right)\right\}_{n=1}^{\infty}$ form a Riesz basis for $\mathcal{H}_{K}$; the sequence $\left\{T_{n}:=\mathcal{T}_{K}\left(x_{n}\right)\right\}_{n=1}^{\infty}$ is its dual Riesz basis. Expanding any $f \in \mathcal{H}_{K}$ with respect the basis $\left\{S_{n}\right\}_{n=1}^{\infty}$ we obtain

$$
f(z)=\sum_{n=1}^{\infty}\left\langle f, T_{n}\right\rangle_{\mathcal{H}_{K}} S_{n}(z) \quad \text { in } \mathcal{H}_{K} .
$$

Besides,

$$
\left\langle f, T_{n}\right\rangle_{\mathcal{H}_{K}}={\overline{\left\langle x, x_{n}\right\rangle_{\mathcal{H}}}}=\left\langle\frac{K\left(z_{n}\right)}{a_{n}}, x\right\rangle_{\mathcal{H}}=\frac{f\left(z_{n}\right)}{a_{n}} .
$$

Since a Riesz basis is an unconditional basis, the sampling series will be pointwise unconditionally convergent and hence, absolutely convergent. The uniform convergence is a standard result in the setting of the RKHS theory since $z \mapsto\|K(z)\|_{\mathcal{H}}$ is bounded on compact subsets of $\mathbb{C}$. Thus we have proved an abstract version of the classical Kramer sampling theorem [16]:

Theorem 1. Let $K: \mathbb{C} \longrightarrow \mathcal{H}$ be an analytic Kramer kernel, and assume that the interpolation property (7) holds for some sequences $\left\{z_{n}\right\}_{n=1}^{\infty}$ in $\mathbb{C}$ and $\left\{a_{n}\right\}_{n=1}^{\infty}$ in $\mathbb{C} \backslash\{0\}$. Let $\mathcal{H}_{K}$ be the corresponding RKHS of entire functions. Then any $f \in \mathcal{H}_{K}$ can be recovered from the sequence of its samples $\left\{f\left(z_{n}\right)\right\}_{n=1}^{\infty}$ by means of the sampling series

$$
f(z)=\sum_{n=1}^{\infty} f\left(z_{n}\right) \frac{S_{n}(z)}{a_{n}}, \quad z \in \mathbb{C} .
$$

This series converges absolutely and uniformly on compact subsets of $\mathbb{C}$. 
Equivalently, the Kramer property in Definition 1 can be seen as a sequence $\left\{z_{n}\right\}_{n=1}^{\infty}$ in $\mathbb{C}$ such that the sequence of reproducing kernels $\left\{k\left(\cdot, z_{n}\right)\right\}_{n=1}^{\infty}$ is a Riesz basis for $\mathcal{H}_{K}$. An interesting problem is to characterize the sequences $\left\{z_{n}\right\}_{n=1}^{\infty}$ in $\mathbb{C}$ having this property in some structured RKHS spaces of entire functions like Hardy or Bergman spaces (see, for instance, Refs. $[2,3,25]$ and references therein).

Concerning the sampling formula (8) in $\mathcal{H}_{K}$, a challenging problem is to give a necessary and sufficient condition to ensure that it can be written as a Lagrange-type interpolation series (see, Eq. (9) below). As it was pointed out in the introduction, it concerns the stability of the functions belonging to the space $\mathcal{H}_{K}$ on removing a finite number of their zeros; it will be called the zero-removing property:

Definition 2. A set $\mathcal{A}$ of entire functions has the zero-removing property (ZR property in short) if for any $g \in \mathcal{A}$ and any zero $w$ of $g$ the function $g(z) /(z-w)$ belongs to $\mathcal{A}$.

A set $\mathcal{A}$ of entire functions has the zero-removing property at a point $a \in \mathbb{C}\left(\mathrm{ZR}_{a}\right.$ property in short) if for any $g \in \mathcal{A}$ with $g(a)=0$ the function $g(z) /(z-a)$ belongs to $\mathcal{A}$.

In fact, the following result holds (see $[8,9]$ for the proof):

Theorem 2. Let $\mathcal{H}_{K}$ be a RKHS of entire functions obtained from an analytic Kramer kernel $K$ with respect to the data $\left\{z_{n}\right\}_{n=1}^{\infty} \subset \mathbb{C}$ and $\left\{a_{n}\right\}_{n=1}^{\infty} \in \mathbb{C} \backslash\{0\}$, i.e., $K\left(z_{n}\right)=a_{n} x_{n}, n \in \mathbb{N}$, for some Riesz basis $\left\{x_{n}\right\}_{n=1}^{\infty}$ for $\mathcal{H}$. Then, the sampling formula (8) for $\mathcal{H}_{K}$ can be written as a Lagrange-type interpolation series

$$
f(z)=\sum_{n=1}^{\infty} f\left(z_{n}\right) \frac{P(z)}{\left(z-z_{n}\right) P^{\prime}\left(z_{n}\right)}, \quad z \in \mathbb{C},
$$

where $P$ denotes an entire function having only simple zeros at $\left\{z_{n}\right\}_{n=1}^{\infty}$ if and only if the space $\mathcal{H}_{K}$ satisfies the $Z R$ property.

The ZR property (also called the division property; see [11]) is ubiquitous in mathematics; for instance, the set $\mathcal{P}_{N}(\mathbb{C})$ of polynomials with complex coefficients of degree less or equal than $N$ has the ZR property. Another more involved examples sharing this property are:

(a) The entire functions in the Pólya class have the ZR property $[4, \mathrm{p} .15]$. Recall that an entire function $E(z)$ is said to be of Pólya class if it has no zeros in the upper half-plane, if $|E(x-i y)| \leq|E(x+i y)|$ for $y>0$, and if $|E(x+i y)|$ is a nondecreasing function of $y>0$ for each fixed $x$.

(b) The Paley-Wiener space $P W_{\pi}$ satisfies the ZR property; it follows immediately from its characterization as the space of entire functions $f$ such that $|f(z)| \leq A \mathrm{e}^{\pi|z|}$ on $\mathbb{C}$ for some positive constant $A$, and $\left.f\right|_{\mathbb{R}} \in L^{2}(\mathbb{R})$, i.e., the classical Paley-Wiener theorem [28, p. 85]. For a direct proof, consider $f \in P W_{\pi}$ such that $f(a)=0$, i.e., 


$$
f(z)=\frac{1}{\sqrt{2 \pi}} \int_{-\pi}^{\pi} \mathrm{e}^{i w z} \widehat{f}(w) d w, z \in \mathbb{C}, \text { such that } \int_{-\pi}^{\pi} \mathrm{e}^{i w a} \widehat{f}(w) d w=0
$$

where $\widehat{f}$ stands for the Fourier transform of $f$. Consider the function $g(w)=$ $\int_{-\pi}^{w} \mathrm{e}^{i a x} \widehat{f}(x) d x$ which satisfies $g(-\pi)=g(\pi)=0$. Integrating by parts one obtains

$$
\frac{f(z)}{z-a}=\frac{1}{\sqrt{2 \pi}} \frac{1}{z-a} \int_{-\pi}^{\pi} \mathrm{e}^{i(z-a) w} \mathrm{e}^{i w a} \widehat{f}(w) d w=\frac{-1}{\sqrt{2 \pi}} \int_{-\pi}^{\pi} \mathrm{e}^{i w z}\left(i \mathrm{e}^{-i a w} g(w)\right) d w .
$$

In other words, since the function $-i \mathrm{e}^{-i a w} g(w)$ belongs to $L^{2}[-\pi, \pi]$, the function $f(z) /(z-a)$ belongs to $P W_{\pi}$.

(c) In general, a de Branges space $\mathcal{H}(E)$ with strict de Branges (structure) function $E$ has the ZR property [4, p. 52]. Let $E$ be an entire function verifying $|E(x-i y)|<|E(x+i y)|$ for all $y>0$. The de Branges space $\mathcal{H}(E)$ is the set of all entire functions $f$ such that

$$
\|f\|_{E}^{2}:=\int_{-\infty}^{\infty}\left|\frac{f(t)}{E(t)}\right|^{2} d t<\infty
$$

and such that both ratios $f / E$ and $f^{*} / E$, where $f^{*}(z):=\overline{f(\bar{z})}, z \in \mathbb{C}$, are of bounded type and of nonpositive mean type in the upper half-plane. The structure function or de Branges function $E$ has no zeros in the upper half plane. A de Branges function $E$ is said to be strict if it has no zeros on the real axis. We require $f / E$ and $f^{*} / E$ to be of bounded type and nonpositive mean type in $\mathbb{C}^{+}$. A function is of bounded type if it can be written as a quotient of two bounded analytic functions in $\mathbb{C}^{+}$and it is of nonpositive mean type if it grows no faster than $\mathrm{e}^{\varepsilon y}$ for each $\varepsilon>0$ as $y \rightarrow \infty$ on the positive imaginary axis $\{$ iy : $y>0\}$. Note that the Paley-Wiener space $P W_{\pi}$ is a de Branges space with strict structure function $E_{\pi}(z)=\exp (-\mathrm{i} \pi z)$.

As a consequence of Theorem 2, any sampling formula like Eq. 8 in a de Branges space can be written as a Lagrange-type interpolation series.

(d) Whenever the space $\mathcal{H}_{K}$ is associated with a polynomial kernel $K(z):=$ $\sum_{n=0}^{N} c_{n} z^{n}$, where $c_{n} \in \mathcal{H}$ and $c_{N} \neq 0$, it is easy to give a characterization for the ZR property in $\mathcal{H}_{K}$. Namely, the ZR property holds in $\mathcal{H}_{K}$ if and only if the set $\left\{c_{0}, c_{1}, \ldots, c_{N}\right\}$ is linearly independent in $\mathcal{H}$ (see [9] for a proof). A more involved problem is to deal with a general entire $\mathcal{H}$-valued kernel $K(z)=\sum_{n=0}^{\infty} c_{n} z^{n}, z \in \mathbb{C}$; the aim of this paper is to obtain some results in this direction.

(e) In a separable Hilbert space $\mathcal{H}$ with orthonormal basis $\left\{e_{n}\right\}_{n=0}^{\infty}$ consider the kernel

$$
\begin{aligned}
K_{\gamma}: \mathbb{C} & \longrightarrow \mathcal{H} \\
& z \longmapsto K_{\gamma}(z):=\sum_{n=0}^{\infty} \frac{e_{n}}{\gamma_{n}} z^{n}
\end{aligned}
$$


where $\gamma:=\left\{\gamma_{n}\right\}_{n=0}^{\infty}$ is a sequence of positive real numbers such that the sequence of quotients $\left\{\gamma_{n} / \gamma_{n+1}\right\}_{n \in \mathbb{N}_{0}}$ decreases to zero as $n$ increases to infinity. The corresponding spaces $\mathcal{H}_{K_{\gamma}}$ constructed from this family of analytic kernels were introduced by Chan and Shapiro in [5]. Obviously, an entire function $f(z)=\sum_{n=0}^{\infty} \alpha_{n} z^{n}$ belongs to the space $\mathcal{H}_{K_{\gamma}}$ if and only if the sequence $\left\{\gamma_{n} \alpha_{n}\right\}_{n \in \mathbb{N}_{0}}$ belongs to $\ell^{2}\left(\mathbb{N}_{0}\right)$, where, as usual, $\mathbb{N}_{0}:=\mathbb{N} \cup\{0\}$. Therefore, it is straightforward to show that if $f \in \mathcal{H}_{K_{\gamma}}$, with $f(0)=0$, then $f(z) / z$ belongs to $\mathcal{H}_{K_{\gamma}}$, i.e., the space $\mathcal{H}_{K_{\gamma}}$ satisfies the $\mathrm{ZR}_{0}$ property. If the sequence $\left\{\gamma_{n+1} / \gamma_{n}\right\}_{n \in \mathbb{N}_{0}}$ is $O(1 / n)$ as $n \rightarrow \infty$, then, for any $a \in \mathbb{C}$, the translation operator given by $T_{a} f(z):=f(z-a), z \in \mathbb{C}$, is a well-defined bounded operator $T_{a}: \mathcal{H}_{K_{\gamma}} \longrightarrow \mathcal{H}_{K_{\gamma}}$ (see [5] for the details). As a consequence of this fact, the space $\mathcal{H}_{K_{\gamma}}$ satisfies the ZR property (for the details, see Eq. (20) below).

Next we include some examples of spaces $\mathcal{H}_{K}$ where the ZR property fails:

(f) Let $K: \mathbb{C} \rightarrow \mathcal{H}$ be an analytic kernel and assume that there exist two distinct points $z_{1}$ and $z_{2} \in \mathbb{C}$ such that $K\left(z_{1}\right)=K\left(z_{2}\right)$. Then the space $\mathcal{H}_{K}$ does not hold the ZR property. Indeed, for $x \neq 0$ in $\mathcal{H}$, orthogonal to $K\left(z_{1}\right)$, consider the function $f(z)=\langle K(z), x\rangle, z \in \mathbb{C}$. Assume that $r$ is the order of the zero $z_{1}$ of $f$. If the property ZR holds in $\mathcal{H}_{K}$, the function

$$
g(z)=\frac{f(z)}{\left(z-z_{1}\right)^{r}}, \quad z \in \mathbb{C}
$$

belongs to $\mathcal{H}_{K}$, and $g\left(z_{1}\right) \neq 0$. Let $y \in \mathcal{H}$ be such that $g(z)=\langle K(z), y\rangle, z \in \mathbb{C}$. Since $g\left(z_{2}\right)=0$ we have that $y$ is orthogonal to $K\left(z_{2}\right)$; but $g\left(z_{1}\right) \neq 0$ implies that $y$ is not orthogonal to $K\left(z_{1}\right)$, that is, a contradiction.

(g) Finally, we exhibit a nontrivial example taken from [9] of a RKHS $\mathcal{H}_{K}$, built from the Sobolev Hilbert space $\mathcal{H}:=H^{1}(-\pi, \pi)$, where the ZR property fails. Namely: consider the Sobolev Hilbert space $H^{1}(-\pi, \pi)$ with its usual inner product

$$
\langle f, g\rangle_{1}=\int_{-\pi}^{\pi} f(x) \overline{g(x)} d x+\int_{-\pi}^{\pi} f^{\prime}(x) \overline{g^{\prime}(x)} d x, \quad f, g \in H^{1}(-\pi, \pi) .
$$

The sequence $\left\{e^{i n x}\right\}_{n \in \mathbb{Z}} \cup\{\sinh x\}$ forms an orthogonal basis for $H^{1}$ $(-\pi, \pi)$ : It is straightforward to prove that the orthogonal complement of $\left\{e^{i n x}\right\}_{n \in \mathbb{Z}}$ in $H^{1}(-\pi, \pi)$ is a one-dimensional space for which $\sinh x$ is a basis. For a fixed $a \in \mathbb{C} \backslash \mathbb{Z}$ we define a kernel

$$
\begin{aligned}
K_{a}: & \mathbb{C} \longrightarrow H^{1}(-\pi, \pi) \\
z & \longmapsto K_{a}(z),
\end{aligned}
$$

by setting

$$
\left[K_{a}(z)\right](x)=(z-a) e^{i z x}+\sin \pi z \sinh x \quad \text { for } x \in(-\pi, \pi) .
$$


Clearly, $K_{a}$ defines an analytic Kramer kernel. Expanding $K_{a}(z) \in H^{1}$ $(-\pi, \pi)$ in the former orthogonal basis we obtain

$$
\begin{aligned}
& K_{a}(z)=[1-i(z-a)] \sin \pi z \sinh x+(z-a) \sum_{n=-\infty}^{\infty} \frac{1+z n}{1+n^{2}} \\
& \quad \operatorname{sinc}(z-n) e^{i n x} \text { in } H^{1}(-\pi, \pi) .
\end{aligned}
$$

where sinc denotes the cardinal sine function $\operatorname{sinc}(z)=\sin \pi z / \pi z$, if $z \neq 0$, and $\operatorname{sinc}(0)=1$. As a consequence, Theorem 1 gives the following sampling result in $\mathcal{H}_{K_{a}}$ : Any function $f \in \mathcal{H}_{K_{a}}$ can be recovered from its samples $\{f(a)\} \cup\{f(n)\}_{n \in \mathbb{Z}}$ by means of the sampling formula

$$
\begin{aligned}
f(z)= & {[1-i(z-a)] \frac{\sin \pi z}{\sin \pi a} f(a) } \\
& +\sum_{n=-\infty}^{\infty} f(n) \frac{z-a}{n-a} \frac{1+z n}{1+n^{2}} \operatorname{sinc}(z-n), \quad z \in \mathbb{C} .
\end{aligned}
$$

The function $(z-a) \operatorname{sinc} z$ belongs to $\mathcal{H}_{K_{a}}$ since $(z-a)$ sinc $z=\left\langle K_{a}(z), 1 / 2 \pi\right\rangle_{1}$ for each $z \in \mathbb{C}$. However, by using the above sampling formula for $\mathcal{H}_{K_{a}}$ it is straightforward to check that the function sinc $z$ does not belong to $\mathcal{H}_{K_{a}}$. Analogously, one can prove that the zero-removing property also fails for any $n \in \mathbb{Z}$ by considering the function $f(z)=\sin \pi z$ which belongs to $\mathcal{H}_{K_{a}}$.

\section{Some Properties on $\mathcal{H}_{K}$ Related to the Kernel $\boldsymbol{K}$}

In this section we obtain some properties of the Hilbert space $\mathcal{H}_{K}$ derived from the sequence of Taylor coefficients of the entire kernel $K$ at a point $a \in \mathbb{C}$. Indeed, for each $a \in \mathbb{C}$ we have the Taylor expansion

$$
K(z)=\sum_{n=0}^{\infty} c_{n}(a)(z-a)^{n}, \quad z \in \mathbb{C},
$$

where the coefficient $c_{n}(a) \in \mathcal{H}$ for each $n \in \mathbb{N}_{0}$. By using Cauchy's integral formula for derivatives (see [26, p. 268] we have

$$
c_{n}(a)=\frac{1}{n !} K^{(n)}(a)=\frac{1}{2 \pi i} \int_{|z-a|=R} \frac{K(z)}{(z-a)^{n+1}} d z, \quad n=0,1, \ldots,
$$

from which

$$
\left\|c_{n}(a)\right\|_{\mathcal{H}} \leq \frac{1}{R^{n+1}} \sup _{|z-a|=R}\|K(z)\|_{\mathcal{H}}=\frac{M_{R}(a)}{R^{n+1}}
$$

where $M_{R}(a):=\sup _{|z-a|=R}\|K(z)\|_{\mathcal{H}}$. Taking $R>1$, the above inequality shows that the sequence $\left\{\left\|c_{n}(a)\right\|\right\}_{n \in \mathbb{N}_{0}}$ belongs to $\ell^{1}\left(\mathbb{N}_{0}\right) \subset \ell^{2}\left(\mathbb{N}_{0}\right)$.

Proposition 1. Let $\left\{c_{n}(a)\right\}_{n \in \mathbb{N}_{0}}$ be the sequence of Taylor coefficients of $K$ at any $a \in \mathbb{C}$. 
1. The sequence $\left\{c_{n}(a)\right\}_{n \in \mathbb{N}_{0}}$ is a Bessel sequence for $\mathcal{H}$.

2. Assume that the mapping $\mathcal{T}_{K}$ in (4) is injective. Then the sequence $\left\{c_{n}(a)\right\}_{n \in \mathbb{N}_{0}}$ is a complete sequence in $\mathcal{H}$.

Proof. For any $x \in \mathcal{H}$ we have $\left|\left\langle c_{n}(a), x\right\rangle\right|^{2} \leq\left\|c_{n}(a)\right\|_{\mathcal{H}}^{2}\|x\|_{\mathcal{H}}^{2}$ for each $n \in \mathbb{N}_{0}$. Thus, having in mind (10) we obtain

$$
\sum_{n=0}^{\infty}\left|\left\langle c_{n}(a), x\right\rangle\right|^{2} \leq\left(\sum_{n=0}^{\infty}\left\|c_{n}(a)\right\|_{\mathcal{H}}^{2}\right)\|x\|_{\mathcal{H}}^{2} \leq B\|x\|_{\mathcal{H}}^{2},
$$

where $B:=\frac{M_{R}^{2}(a)}{R^{2}-1}$ and $R>1$.

Assume now that $\left\langle c_{n}(a), x\right\rangle=0$ for all $n \in \mathbb{N}_{0}$. For the function $f(z):=$ $\langle K(z), x\rangle, z \in \mathbb{C}$, we have the Taylor expansion

$$
f(z)=\sum_{n=0}^{\infty}\left\langle c_{n}(a), x\right\rangle(z-a)^{n}=0 \quad \text { for all } z \in \mathbb{C} .
$$

Since the anti-linear mapping $\mathcal{T}_{K}$ is injective we deduce that $x=0$.

The Bessel property in Proposition 1 implies that the space $\mathcal{H}_{K}$ is a subspace of the Hardy space $H^{2}(\mathbb{D})$ with continuous inclusion (see [22]). It will be a closed subspace if and only if the sequence $\left\{c_{n}(0)\right\}_{n \in \mathbb{N}_{0}}$ is a frame for $\mathcal{H}$ (see, for instance, $[6,23])$. In this paper we often assume that the sequence $\left\{c_{n}(0)\right\}_{n \in \mathbb{N}_{0}}$ is also minimal (see Definition 3 below); as a consequence, $\left\{c_{n}(0)\right\}_{n \in \mathbb{N}_{0}}$ is a Riesz basis where necessarily $0<m \leq\left\|c_{n}(0)\right\| \leq M<\infty$ for all $n \in \mathbb{N}_{0}$ (see $[6$, p. 124]). This is not the case in our setting since the sequence of Taylor coefficients $c_{n}(0) \rightarrow 0$ in $\mathcal{H}$ as $n \rightarrow \infty$. In other words, the space $\mathcal{H}_{K}$ is not, in general, a closed subspace of the Hardy space $H^{2}(\mathbb{D})$.

As it was mentioned in Sect. 2, whenever $K$ is a polynomial kernel with coefficients in $\mathcal{H}$, a necessary and sufficient condition for $\mathcal{H}_{K}$ satisfying the ZR property is the linear independence in $\mathcal{H}$ of the coefficients of $K$. In the general case, the linear independence of the Taylor coefficients $\left\{c_{n}(0)\right\}_{n \in \mathbb{N}_{0}}$ of $K$ at 0 is only a necessary condition for the $Z_{0}$ property (clearly it is not a sufficient condition; see, for instance, example (g) in Sect. 2):

Proposition 2. Assume that the space $\mathcal{H}_{K}$ satisfies the $Z R_{0}$ property and consider the Taylor expansion $K(z)=\sum_{n=0}^{\infty} c_{n}(0) z^{n}$ of $K$ around 0 . Then, the sequence $\left\{c_{n}(0)\right\}_{n \in \mathbb{N}_{0}}$ is linearly independent in $\mathcal{H}$.

Proof. Assume that there exists an index $N$ such that the coefficient $c_{N}(0)$ depends linearly on $\left\{c_{0}(0), c_{1}(0), \ldots, c_{N-1}(0)\right\}$, and consider a non-zero $x \in$ $\left\{c_{0}(0), c_{1}(0), \ldots, c_{N-1}(0)\right\}^{\perp}$. Then, the function $\langle K(z), x\rangle$ satisfies

$$
\langle K(z), x\rangle=z^{m}\left(\left\langle c_{m}(0), x\right\rangle+\left\langle c_{m+1}(0), x\right\rangle z+\left\langle c_{m+2}(0), x\right\rangle z^{2}+\cdots\right)
$$

with $m \geq N+1$ and $\left\langle c_{m}(0), x\right\rangle \neq 0$. If $\mathcal{H}_{K}$ satisfies the $\mathrm{ZR}_{0}$ property, then the entire function

$$
g(z)=z^{N}\left(\left\langle c_{m}(0), x\right\rangle+\left\langle c_{m+1}(0), x\right\rangle z+\left\langle c_{m+2}(0), x\right\rangle z^{2}+\cdots\right)
$$


belongs to $\mathcal{H}_{K}$, that is, there exists $y \in \mathcal{H}$ such that

$$
\left\langle c_{0}(0), y\right\rangle=\left\langle c_{1}(0), y\right\rangle=\cdots=\left\langle c_{N-1}(0), y\right\rangle=0
$$

and

$$
\left\langle c_{m+k}(0), x\right\rangle=\left\langle c_{N+k}(0), y\right\rangle \text { for all } k \geq 0 .
$$

Since $c_{N}(0)$ depends linearly on $\left\{c_{0}(0), c_{1}(0), \ldots, c_{N-1}(0)\right\}$ and $\left\langle c_{N}(0), y\right\rangle$ $\neq 0$ we get a contradiction.

As a consequence of the above result, if the space $\mathcal{H}_{K}$ satisfies the $\mathrm{ZR}$ property then, for each $a \in \mathbb{C}$, the sequence $\left\{c_{n}(a)\right\}_{n \in \mathbb{N}_{0}}$ is linearly independent in $\mathcal{H}$. In other words, if there exists $a \in \mathbb{C}$ such that $\left\{c_{n}(a)\right\}_{n \in \mathbb{N}_{0}}$ is linearly dependent in $\mathcal{H}$, then the ZR property does not hold in $\mathcal{H}_{K}$.

A classical problem in a de Branges space $\mathcal{H}(E)$ is to determine when the set of polynomials $\mathcal{P}(\mathbb{C})$ is included in $\mathcal{H}(E)$ (see [1] and references therein). Next, we study the relationship between the set of polynomials $\mathcal{P}(\mathbb{C})$ and our spaces $\mathcal{H}_{K}$ via the Taylor coefficients $\left\{c_{n}(a)\right\}_{n \in \mathbb{N}_{0}}$ of the kernel $K$ at a point $a \in \mathbb{C}$.

Definition 3. A sequence $\left\{c_{n}\right\}_{n \in \mathbb{N}_{0}}$ is said to be minimal in $\mathcal{H}$ if $c_{m} \notin \overline{\text { span }}$ $\left\{c_{n}\right\}_{n \neq m}$ for each $m \in \mathbb{N}_{0}$. A sequence $\left\{c_{n}\right\}_{n \in \mathbb{N}_{0}}$ is said to be supercomplete in $\mathcal{H}$ if the sequence $\left\{c_{n}\right\}_{n \geq m}$ is complete in $\mathcal{H}$ for each $m \in \mathbb{N}_{0}$.

Obviously, each minimal sequence $\left\{c_{n}\right\}_{n=0}^{\infty}$ is linearly independent in $\mathcal{H}$. In this section we will assume that the mapping $\mathcal{T}_{K}$ in $(4)$ is injective; consequently, the sequence $\left\{c_{n}(a)\right\}_{n \in \mathbb{N}_{0}}$ of Taylor coefficients of $K$ at any $a \in \mathbb{C}$ is a complete sequence in $\mathcal{H}$ (see Proposition 1 ).

Proposition 3. The set of polynomials $\mathcal{P}(\mathbb{C})$ is contained in $\mathcal{H}_{K}$ if and only if the sequence $\left\{c_{n}(0)\right\}_{n \in \mathbb{N}_{0}}$ of Taylor coefficients of $K$ at 0 is minimal in $\mathcal{H}$. Moreover, the sequence $\left\{c_{n}(0)\right\}_{n \in \mathbb{N}_{0}}$ is minimal in $\mathcal{H}$ if and only if the sequence $\left\{c_{n}(a)\right\}_{n \in \mathbb{N}_{0}}$ is minimal in $\mathcal{H}$ for each $a \in \mathbb{C}$.

Proof. For each $n \in \mathbb{N}_{0}$ the monomial $z^{n}$ belongs to $\mathcal{H}_{K}$ if and only if there exists $x_{n} \in \mathcal{H}$ such that $\left\langle c_{m}(0), x_{n}\right\rangle=\delta_{m, n}$, where $\delta_{m, n}$ denotes the Kronecker delta. Equivalently, $\left\{z^{n}\right\}_{n=0}^{\infty} \subset \mathcal{H}_{K}$ if and only if there exists a biorthogonal sequence $\left\{x_{n}\right\}_{n=0}^{\infty} \subset \mathcal{H}$ of $\left\{c_{n}(0)\right\}_{n \in \mathbb{N}_{0}}$. This is known to be equivalent to the minimality of $\left\{c_{n}(0)\right\}_{n \in \mathbb{N}_{0}}$ (see $[28]$ ).

Now, suppose that for some $a \in \mathbb{C}$ the sequence $\left\{c_{n}(a)\right\}_{n \in \mathbb{N}_{0}}$ fails to be minimal. Then there exists $N \in \mathbb{N}_{0}$ such that

$$
c_{N}(a) \in \overline{\operatorname{span}}\left\{c_{0}(a), \ldots, c_{N-1}(a), c_{N+1}(a), \ldots\right\}
$$

Having in mind the completeness of the sequence $\left\{c_{n}(a)\right\}_{n \in \mathbb{N}_{0}}$ in $\mathcal{H}$ we deduce that the sequence $\left\{c_{0}(a), \ldots, c_{N-1}(a), c_{N+1}(a), \ldots\right\}$ is complete in $\mathcal{H}$. Therefore, if $x \in\left\{c_{m}\right\}_{m \neq N}^{\perp}$ then $x=0$ and, consequently, the polynomial $(z-a)^{N}$ does not belong to $\mathcal{H}_{K}$. 
Proposition 4. The sequence $\left\{c_{n}(0)\right\}_{n \in \mathbb{N}_{0}}$ of Taylor coefficients of $K$ at 0 is supercomplete in $\mathcal{H}$ if and only if the space $\mathcal{H}_{K}$ does not contain any non-zero polynomial. Moreover, the sequence $\left\{c_{n}(0)\right\}_{n \in \mathbb{N}_{0}}$ is supercomplete in $\mathcal{H}$ if and only if the sequence $\left\{c_{n}(a)\right\}_{n \in \mathbb{N}_{0}}$ is supercomplete in $\mathcal{H}$ for each $a \in \mathbb{C}$.

Proof. A non-zero polynomial $a_{N} z^{N}+a_{N-1} z^{N-1}+\cdots+a_{0}$ belongs to $\mathcal{H}_{K}$ if and only if there exists $x \in \mathcal{H}, x \neq 0$, such that

$$
\begin{aligned}
\left\langle c_{0}(0), x\right\rangle & =a_{0},\left\langle c_{1}(0), x\right\rangle=a_{1}, \ldots,\left\langle c_{N}(0), x\right\rangle \\
& =a_{N} \text { and }\left\langle c_{m}(0), x\right\rangle=0 \quad \text { for } m>N .
\end{aligned}
$$

Hence, a non-zero polynomial is in $\mathcal{H}_{K}$ if and only if the sequence $\left\{c_{n}(0)\right\}_{n \in \mathbb{N}_{0}}$ is not supercomplete in $\mathcal{H}$.

Now, suppose that the sequence $\left\{c_{n}(0)\right\}_{n \in \mathbb{N}_{0}}$ is supercomplete in $\mathcal{H}$ and that, for some $b \in \mathbb{C}$, the sequence $\left\{c_{n}(b)\right\}_{n \in \mathbb{N}_{0}}$ is not supercomplete in $\mathcal{H}$. Then, there exists $N \in \mathbb{N}_{0}$ such that sequence $\left\{c_{N+1}(b), c_{N+2}(b), \ldots\right\}$ is not complete in $\mathcal{H}$. Therefore, there exists a non-zero $x \in \mathcal{H}$ such that $\left\langle c_{m}(b), x\right\rangle=$ 0 for all $m>N$. As a consequence, the non-zero polynomial

$$
\left\langle c_{0}(b), x\right\rangle+\left\langle c_{1}(b), x\right\rangle(z-b)+\cdots+\left\langle c_{N}(b), x\right\rangle(z-b)^{N}
$$

belongs to $\mathcal{H}_{K}$, that is, a contradiction.

In the Paley-Wiener case, the Fourier kernel $K(z)(w)=\frac{1}{\sqrt{2 \pi}} e^{i z w}, w \in$ $[-\pi, \pi]$, can be expanded, around $a \in \mathbb{C}$, as

$$
K(z)(w)=\frac{1}{\sqrt{2 \pi}} e^{i z w}=\frac{1}{\sqrt{2 \pi}} \sum_{n=0}^{\infty} e^{i a w} \frac{(i w)^{n}}{n !}(z-a)^{n}, \quad z \in \mathbb{C} .
$$

Hence, for $n \in \mathbb{N}_{0}$, we get that $c_{n}(0)(w)=\frac{1}{\sqrt{2 \pi}} \frac{(i w)^{n}}{n !}, w \in[-\pi, \pi]$.

As a by-product, since the Paley-Wiener space $P W_{\pi}$ does not contain any non-zero polynomial, from Proposition 4 we deduce that the sequence of monomials $\left\{1, w, w^{2}, \ldots\right\}$ is supercomplete (and hence, it is not minimal) in $L^{2}[-\pi, \pi]$.

Concerning the $\mathrm{ZR}$ property in $\mathcal{H}_{K}$ and the relationship between the set $\mathcal{P}(\mathbb{C})$ of polynomials and $\mathcal{H}_{K}$ we have the following result:

Proposition 5. Suppose that the space $\mathcal{H}_{K}$ satisfies the $Z R$ property. Then, only one of the following three cases hold:

(a) For any $a \in \mathbb{C}$ the sequence $\left\{c_{n}(a)\right\}_{n \in \mathbb{N}_{0}}$ is minimal in $\mathcal{H}$. In this case the space $\mathcal{H}_{K}$ contains any polynomial.

(b) For any $a \in \mathbb{C}$ the sequence $\left\{c_{n}(a)\right\}_{n \in \mathbb{N}_{0}}$ is supercomplete in $\mathcal{H}$. In this case the space $\mathcal{H}_{K}$ does not contain non-zero polynomials.

(c) There exists $N \in \mathbb{N}_{0}$ such that the polynomials belonging to $\mathcal{H}_{K}$ are precisely the set of polynomials of degree less or equal than $N$. In this case, for each $a \in \mathbb{C}$ the sequence $\left\{c_{n}(a)\right\}_{n \geq N+1}$ is supercomplete in its closed span and $c_{r}(a) \notin \overline{\operatorname{span}}\left\{c_{n}(a)\right\}_{n \neq r}$ for $r=0,1, \ldots, N$. 
Proof. We denote by $\partial p$ the degree of a polynomial $p$. Assume that there exists a polynomial $p$ belonging to the space $\mathcal{H}_{K}$. If the space $\mathcal{H}_{K}$ satisfies the ZR property then the set of polynomials whose degree is less or equal than $\partial p$ is included in $\mathcal{H}_{K}$.

If the case a. does not hold, consider $N:=\max _{r \in \mathbb{N}_{0}}\left\{q \in \mathcal{H}_{K} \mid q\right.$ polynomial and $\partial q=r\}$ which is finite. Since the ZR property holds, the set of polynomials of degree less or equal than $N$ is included in $\mathcal{H}_{K}$.

\section{The Zero-Removing Property at a Fixed Point}

In this section we study conditions under which, for a fixed $a \in \mathbb{C}$, the $\mathrm{ZR}_{a}$ property holds in $\mathcal{H}_{K}$. Reducing the $\mathrm{ZR}_{a}$ property to a moment problem, a sufficient condition assuring that the $\mathrm{ZR}_{a}$ property holds involves the continuity of a shift-type operator.

\subsection{A Sufficient Condition for the $\mathbf{Z R}_{a}$ Property}

Consider a function $f \in \mathcal{H}_{K}$, i.e., $f(z)=\langle K(z), x\rangle_{\mathcal{H}}$ on $\mathbb{C}$ for some $x \in \mathcal{H}$, such that $f(a)=0$. Then $\left\langle c_{0}(a), x\right\rangle=0$ and

$$
\frac{f(z)}{z-a}=\sum_{n=0}^{\infty}\left\langle c_{n+1}(a), x\right\rangle(z-a)^{n}, \quad z \in \mathbb{C} .
$$

As a consequence, the space $\mathcal{H}_{K}$ satisfies the property $\mathrm{ZR}_{a}$ if and only if for each $x \in\left\{c_{0}(a)\right\}^{\perp}$ there exists $y \in \mathcal{H}$ such that

$$
\left\langle c_{n}(a), y\right\rangle=\left\langle c_{n+1}(a), x\right\rangle, \quad n \in \mathbb{N}_{0} .
$$

For the sake of completeness we include the following result on general moment problems whose proof can be found in [28, p. 126]:

Theorem 3. Let $\left\{f_{1}, f_{2}, f_{3}, \ldots\right\}$ be a sequence of vectors belonging to a Hilbert space $\mathcal{H}$ and $\left\{d_{1}, d_{2}, d_{3}, \ldots\right\}$ a sequence of scalars. In order that the equations

$$
\left\langle f, f_{n}\right\rangle=d_{n}, \quad n \in \mathbb{N}
$$

shall admit at least one solution $f \in \mathcal{H}$ for which $\|f\| \leq M$, it is necessary and sufficient that

$$
\left|\sum_{n} a_{n} \bar{d}_{n}\right| \leq M\left\|\sum_{n} a_{n} f_{n}\right\|
$$

for every finite sequence of scalars $\left\{a_{n}\right\}$. If the sequence $\left\{f_{1}, f_{2}, f_{3}, \ldots\right\}$ is complete in $\mathcal{H}$, then the solution is unique.

As a consequence of the above result we obtain: 
Proposition 6. The space $\mathcal{H}_{K}$ satisfies the $Z R_{a}$ property if and only if for each $x \in\left\{c_{0}(a)\right\}^{\perp}$ the linear functional $\mu_{a, x}$ defined on $Y_{a}:=\operatorname{span}\left\{c_{n}(a)\right\}_{n \in \mathbb{N}_{0}}$ as

$$
\mu_{a, x}\left(\sum_{n} a_{n} c_{n}(a)\right)=\sum_{n} a_{n}\left\langle c_{n+1}(a), x\right\rangle
$$

for every finite sequence of scalars $\left\{a_{n}\right\}$, is bounded.

Assume that the sequence $\left\{c_{n}(a)\right\}_{n \in \mathbb{N}_{0}}$ is linearly independent. The linear functional $\mu_{a, x}: Y_{a} \rightarrow \mathbb{C}$ can be decomposed as $T_{a, x} \circ S_{a}$ where $T_{a, x}: Y_{a} \rightarrow \mathbb{C}$ is the linear operator given by

$$
T_{a, x}\left(\sum_{n} a_{n} c_{n}(a)\right)=\sum_{n} a_{n}\left\langle c_{n}(a), x\right\rangle
$$

and $S_{a}: Y_{a} \rightarrow Y_{a}$, is the linear operator given by

$$
S_{a}\left(\sum a_{n} c_{n}(a)\right)=\sum a_{n} c_{n+1}(a)
$$

for every finite sequence of scalars $\left\{a_{n}\right\}$. Observe that $S_{a}$ is a well-defined linear operator since the sequence $\left\{c_{n}(a)\right\}_{n \in \mathbb{N}_{0}}$ is linearly independent. From now on we will assume that the sequence $\left\{c_{n}(a)\right\}_{n \in \mathbb{N}_{0}}$ is linearly independent (see Proposition 2 above). Note that operator $S_{a}$ is nothing but a generalization of the classical shift operator defined by means of an orthonormal basis [22]; here it is defined by means of a linear independent Bessel sequence in $\mathcal{H}$.

The operator $T_{a, x}$ is obviously bounded since

$$
T_{a, x}\left(\sum_{n} a_{n} c_{n}(a)\right)=\sum_{n} a_{n}\left\langle c_{n}(a), x\right\rangle=\left\langle\sum_{n} a_{n} c_{n}(a), x\right\rangle .
$$

Thus, we have obtained the following result:

Theorem 4. Assume that, for each $a \in \mathbb{C}$, the sequence $\left\{c_{n}(a)\right\}_{n \in \mathbb{N}_{0}}$ is linearly independent and the corresponding operator $S_{a}$ given by $(11)$ is bounded. Then the space $\mathcal{H}_{K}$ satisfies the $Z R$ property.

Notice that, for the Paley-Wiener space $P W_{\pi}$, the corresponding operator $S_{a}$ is bounded for any $a \in \mathbb{C}$. Indeed, for $a=0$, we have that $\frac{d}{d w} c_{n+1}(0)(w)$ $=i c_{n}(0)(w), w \in(-\pi, \pi)$, from which we deduce that

$$
S_{0} f(w):=i \int_{0}^{w} f(s) d s \quad \text { for any } f \in L^{2}[-\pi, \pi] .
$$

Since $\left\|S_{0} f\right\|_{2} \leq 2 \pi\|f\|_{2}$ for any $f \in L^{2}[-\pi, \pi], S_{0}$ is bounded on span $\left\{c_{n}(0)\right\}_{n \in \mathbb{N}_{0}}=L^{2}[-\pi, \pi]$. For a non-zero $a \in \mathbb{C}$ we have that $S_{a} f(w)=$ $\mathrm{e}^{i a w} S_{0} f(w)$ and, as a consequence, the operator $S_{a}$ is bounded for each $a \in \mathbb{C}$.

The reciprocal of Theorem 4 remains true under the hypothesis that the function $1 \in \mathcal{H}_{K}$. 
Theorem 5. Assume that the mapping $\mathcal{T}_{K}$ in (4) is injective, the sequence $\left\{c_{n}(a)\right\}_{n=1}^{\infty}$ is linearly independent for any $a \in \mathbb{C}$, and $1 \in \mathcal{H}_{K}$. Then, the space $\mathcal{H}_{K}$ satisfies the $Z R$ property if and only if the operator $S_{a}$ is bounded for each $a \in \mathbb{C}$.

Proof. From Theorem 4 it is enough to show that if $1 \in \mathcal{H}_{K}$ and the $\mathrm{ZR}_{a}$ property holds, then the operator $S_{a}$ is bounded. Let $\sum_{n=0}^{L} a_{n} c_{n}(a)$ be a vector in $Y_{a}$. We have,

$$
\begin{aligned}
\left|\mu_{a, x}\left(\sum_{n=0}^{L} a_{n} c_{n}(a)\right)\right| & =\left|\left\langle\sum_{n=0}^{L} a_{n} c_{n+1}(a), x\right\rangle\right| \\
& =\left\|S_{a}\left(\sum_{n=0}^{L} a_{n} c_{n}(a)\right)\right\|\|x\|\left|\cos \left(\sum_{n=0}^{L} a_{n} c_{n+1}(a), x\right)\right|
\end{aligned}
$$

First we prove that the function $1 \in \mathcal{H}_{K}$ if and only if the condition $c_{0}(a) \notin \overline{\operatorname{span}}\left\{c_{n}(a)\right\}_{n=1}^{\infty}$ holds for each $a \in \mathbb{C}$. Indeed, $1=\langle K(z), x\rangle$ for some $x \in \mathcal{H}$ (necessarily $x \neq 0$ ) implies that, for each $a \in \mathbb{C},\left\langle c_{n}(a), x\right\rangle=0, n \geq 1$. From the completeness of $\left\{c_{n}(a)\right\}_{n \in \mathbb{N}_{0}}$ (see Proposition 1) we deduce that $c_{0}(a) \notin \overline{\operatorname{span}}\left\{c_{n}(a)\right\}_{n=1}^{\infty}$. For the sufficient condition, let $b \in \mathbb{C}$ such that $c_{0}(b) \notin \overline{\operatorname{span}}\left\{c_{n}(b)\right\}_{n=1}^{\infty}$; there exists $x \neq 0$ in $\left(\left\{c_{n}(b)\right\}_{n=1}^{\infty}\right)^{\perp}$ and, as a consequence, the non-zero constant $\langle K(z), x\rangle$ belongs to $\mathcal{H}_{K}$. Note that the condition $c_{0}(b) \notin \overline{\operatorname{span}}\left\{c_{n}(b)\right\}_{n=1}^{\infty}$ for some $b \in \mathbb{C}$ is equivalent to the condition $c_{0}(a) \notin \overline{\operatorname{span}}\left\{c_{n}(a)\right\}_{n=1}^{\infty}$ for every $a \in \mathbb{C}$. Therefore, the hypothesis $1 \in \mathcal{H}_{K}$ implies the existence of a positive number $\alpha$ such that $|\cos (\widehat{v, x})|=\frac{|\langle v, x\rangle|}{\|v\|\|x\|}>$ $\alpha>0$ for any nonzero $v \in \operatorname{span}\left\{c_{n}(a)\right\}_{n=1}^{\infty}$ and any $x \in c_{0}(a)^{\perp} \backslash\{0\}$. Hence,

$$
\left\|S_{a}\left(\sum_{n=0}^{L} a_{n} c_{n}(a)\right)\right\| \leq \frac{1}{\alpha\|x\|}\left|\mu_{a, x}\left(\sum_{n=0}^{L} a_{n} c_{n}(a)\right)\right| .
$$

Since the $\mathrm{ZR}_{a}$ property holds, the linear functional $\mu_{a, x}$ is bounded in $Y_{a}$. Thus, the inequality (12) implies the boundedness of the operator $S_{a}$ on $Y_{a}$.

In the context of de Branges spaces, Baranov [1], improving a previous work in $[14,15]$, solved the problem of finding the structure functions $E$ of zero exponential type for which $1 \in \mathcal{H}(E)$. Since de Branges spaces are particular cases of $\mathcal{H}_{K}$ spaces [10], the condition $1 \in \mathcal{H}(E)$ could be replaced by the equivalent geometric condition $c_{0}(a) \notin \overline{\operatorname{span}}\left\{c_{n}(a)\right\}_{n=1}^{\infty}$ for some $a \in \mathbb{C}$.

\subsection{A Sufficient Condition for the Global ZR Property in a $\mathcal{H}_{K}$ Space}

In this section we give a sufficient condition on the continuity of the operator, say $S_{0}$, under the assumption of the minimality of the sequence $\left\{c_{n}(0)\right\}_{n \in \mathbb{N}_{0}}$ in $\mathcal{H}$, i.e., any polynomial belongs to $\mathcal{H}_{K}$ (see Proposition 3 ). Following Ref. 
$\left[12\right.$, p. 27], the minimality of the sequence $\left\{c_{n}(0)\right\}_{n \in \mathbb{N}_{0}}$ in $\mathcal{H}$ implies that the numbers $\delta_{k}$ given by

$$
\delta_{k}:=\inf _{\theta \in \mathbb{R}} \rho\left(\mathrm{e}^{i \theta} \frac{c_{k}(0)}{\left\|c_{k}(0)\right\|}, \overline{\operatorname{span}}\left\{c_{n}(0)\right\}_{n \neq k}\right), \quad k \in \mathbb{N}_{0},
$$

are strictly positive for every $k \in \mathbb{N}_{0}$. Note that the number $\delta_{k}$ denotes the inclination in $\mathcal{H}$ of the straight line spanned by $c_{k}(0)$ to the closed subspace $\overline{\operatorname{span}}\left\{c_{n}(0)\right\}_{n \neq k}$, being $\rho$ the distance with respect to the metric given by the norm in $\mathcal{H}$.

Besides (see [12, pp. 27-28]), for any $x=\sum_{k} a_{k} c_{k}(0)$ (finite or convergent sum) the inequalities

$$
\left|a_{k}\right| \leq \frac{\|x\|}{\delta_{k}\left\|c_{k}(0)\right\|} \quad \text { hold for each } k \in \mathbb{N}_{0}
$$

Lemma 6. Assume that the sequence $\left\{c_{n}(0)\right\}_{n \in \mathbb{N}_{0}}$ of Taylor coefficients of $K$ at 0 is complete and minimal in $\mathcal{H}$. The convergence of the series

$$
\sum_{n=0}^{\infty} \frac{1}{\delta_{n}} \frac{\left\|c_{n+1}(0)\right\|}{\left\|c_{n}(0)\right\|}
$$

where the numbers $\delta_{n}>0, n \in \mathbb{N}_{0}$, are given in (13), implies that the operator $S_{0}$ is bounded.

Proof. For any finite sum $x=\sum_{n} a_{n} c_{n}(0)$, using inequalities (14), we have

$$
\left\|S_{0} x\right\| \leq \sum_{n}\left|a_{n}\right|\left\|c_{n+1}(0)\right\| \leq\left(\sum_{n} \frac{1}{\delta_{n}} \frac{\left\|c_{n+1}(0)\right\|}{\left\|c_{n}(0)\right\|}\right)\|x\| \leq M\|x\|,
$$

where $M$ denotes the sum of the series in (15). Therefore, the operator $S_{0}$ is bounded on $\operatorname{span}\left\{c_{n}(0)\right\}_{n \in \mathbb{N}_{0}}$; the completeness of $\left\{c_{n}(0)\right\}_{n \in \mathbb{N}_{0}}$ proves that $S_{0}$ is bounded on $\mathcal{H}$.

In fact, the following result holds:

Theorem 7. Assume that the sequence $\left\{c_{n}(0)\right\}_{n \in \mathbb{N}_{0}}$ of Taylor coefficients of $K$ at 0 is complete and minimal in $\mathcal{H}$. Suppose also that the series in (15) converges and the sequence of quotients $\left\{\left\|c_{n+1}(0)\right\| /\left\|c_{n}(0)\right\|\right\}_{n \in \mathbb{N}_{0}}$ is monotonically decreasing. Then, the $Z R$ property in $\mathcal{H}_{K}$ holds.

Proof. By Lemma 6 the $\mathrm{ZR}_{0}$ property holds. Let $a$ be a nonzero complex number and let $f(z)=\sum_{n=0}^{\infty} \alpha_{n} z^{n} \in \mathcal{H}_{K}$ be such that $f(a)=0$. Then,

$$
g(z)=\frac{f(z)}{z-a}=-\frac{1}{a} \sum_{n=0}^{\infty} c_{n} z^{n} \quad \text { where } \quad c_{n}=\frac{1}{a^{n}} \sum_{k=0}^{n} \alpha_{k} a^{k} .
$$


Since $f(a)=\sum_{k=0}^{\infty} \alpha_{k} a^{k}=0$, we have that $\sum_{k=0}^{n} \alpha_{k} a^{k}=-\sum_{k=n+1}^{\infty} \alpha_{k} a^{k}$. Hence,

$$
g(z)=\sum_{n=0}^{\infty}\left(\frac{1}{a^{n+1}} \sum_{k=n+1}^{\infty} \alpha_{k} a^{k}\right) z^{n}
$$

The entire function $g$ belongs to $\mathcal{H}_{K}$ if and only if the linear functional defined on $Y_{0}=\operatorname{span}\left\{c_{n}(0)\right\}_{n \in \mathbb{N}_{0}}$ as

$$
\nu_{f}\left(\sum_{n} b_{n} c_{n}(0)\right)=\sum_{n} b_{n}\left(\frac{1}{a^{n+1}} \sum_{k=n+1}^{\infty} \alpha_{k} a^{k}\right),
$$

for every finite sequence of scalars $\left\{b_{n}\right\}$, is bounded. For any $y=\sum_{n} b_{n} c_{n}(0)$, using inequalities (14) we have

$$
\left|\nu_{f}(y)\right| \leq\|y\|\|x\| \sum_{n} \frac{\left\|c_{n+1}(0)\right\|}{\delta_{n}\left\|c_{n}(0)\right\|} \sum_{m=0}^{\infty} \frac{\left\|c_{m+n+1}(0)\right\|}{\left\|c_{n+1}(0)\right\|}|a|^{m},
$$

where $f=\mathcal{T}_{K} x$. Applying the ratio test it is straightforward to prove that the series $\sum_{m=0}^{\infty} \frac{\left\|c_{m+n+1}(0)\right\|}{\left\|c_{n+1}(0)\right\|} z^{m}$ defines an entire function $G_{n}$ fon any $n \in \mathbb{N}_{0}$. Moreover, since the sequence $\left\{\left\|c_{l+1}(0)\right\| /\left\|c_{l}(0)\right\|\right\}_{l \in \mathbb{N}_{0}}$ is monotonically decreasing, we have that $G_{n}(|a|) \geq G_{n+1}(|a|)$ for any $a \in \mathbb{C}$. As a consequence, for any $y \in Y_{0}=\operatorname{span}\left\{c_{n}(0)\right\}_{n \in \mathbb{N}_{0}}$ we obtain that

$$
\left|\nu_{f}(y)\right| \leq\left(\|x\| G_{0}(|a|) \sum_{n=0}^{\infty} \frac{\left\|c_{n+1}(0)\right\|}{\delta_{n}\left\|c_{n}(0)\right\|}\right)\|y\|=M_{f, a}\|y\|,
$$

i.e., the boundedness of $\nu_{f}$.

\subsection{On the Local Zero-Removing Property}

In this section we will assume that $S_{0}$ is well-defined bounded operator on $\operatorname{span}\left\{c_{n}(0)\right\}_{n \in \mathbb{N}_{0}}$. As a consequence, the ZR $\mathrm{ZR}_{0}$ property holds in $\mathcal{H}_{K}$. This means that for each function $f \in \mathcal{H}_{K}$ with $f(0)=0$, the function $f(z) / z$ belongs to $\mathcal{H}_{K}$. Our goal here is to prove that the $\mathrm{ZR}_{a}$ property also holds for $a \in \mathbb{C}$ with $|a|$ small enough.

We will also assume that the operator $\mathcal{T}_{K}: \mathcal{H} \longrightarrow \mathcal{H}_{K}$ given in (4) is injective. Therefore, for any $a \in \mathbb{C}$ the sequence $\left\{c_{n}(a)\right\}_{n \in \mathbb{N}_{0}}$ is complete in $\mathcal{H}$. The completeness of the sequence $\left\{c_{n}(0)\right\}_{n \in \mathbb{N}_{0}}$ implies that $S_{0}$ can be extended to $\mathcal{H}$ as a bounded operator. Let $S_{0}^{*}$ be its adjoint bounded operator, i.e., for each $x, y$ in $\mathcal{H}$ we have $\left\langle x, S_{0} y\right\rangle=\left\langle S_{0}^{*} x, y\right\rangle$.

By using the bijective anti-linear isometry $\mathcal{T}_{K}$, we define two bounded operators on $\mathcal{H}_{K}$ as $\mathfrak{S}_{0}=\mathcal{T}_{K} S_{0} \mathcal{T}_{K}^{-1}$ and $\mathfrak{S}_{0}^{*}=\mathcal{T}_{K} S_{0}^{*} \mathcal{T}_{K}^{-1}$.

For each $x \in \mathcal{H}$, having in mind that $K(z)=\sum_{n=0}^{\infty} c_{n}(0) z^{n}$, we have

$$
\left\langle K(z), S_{0}^{*} x\right\rangle=\left\langle S_{0} K(z), x\right\rangle=\sum_{n=0}^{\infty}\left\langle c_{n+1}(0), x\right\rangle z^{n}=\frac{f(z)-f(0)}{z},
$$


being $f(z)=\langle K(z), x\rangle=\sum_{n=0}^{\infty}\left\langle c_{n}(0), x\right\rangle z^{n}$. Since $\mathcal{T}_{K} S_{0}^{*} x=\mathfrak{S}_{0}^{*} \mathcal{T}_{K} x=$ $\mathfrak{S}_{0}^{*}(f)$, we deduce that

$$
\mathfrak{S}_{0}^{*} f(z)=\frac{f(z)-f(0)}{z}, \quad z \in \mathbb{C} .
$$

In general, assuming that $S_{a}$ is bounded, the $\mathrm{ZR}_{a}$ property holds and the bounded operator $\mathfrak{S}_{a}^{*}:=\mathcal{T}_{K} S_{a}^{*} \mathcal{T}_{K}^{-1}$ from $\mathcal{H}_{K} \rightarrow \mathcal{H}_{K}$ satisfies that

$$
\mathfrak{S}_{a}^{*} f(z)=\frac{f(z)-f(a)}{z-a}, \quad z \in \mathbb{C} .
$$

Notice that in the de Branges spaces theory, a natural question is whether the space is closed under forming difference quotients as in (16), which means that the function 1 is an associated function (see, for instance, [4,27]).

For each $a \in \mathbb{C}$ we denote by $\mathcal{H}_{a}$ the set

$$
\mathcal{H}_{a}:=\left\{f \in \mathcal{H}_{K} \text { such that } f(a)=0\right\} .
$$

It is straightforward to prove that $\mathcal{H}_{a}$ is a closed subspace of $\mathcal{H}_{K}$ for each $a \in \mathbb{C}$. Indeed, Let $\left\{f_{n}\right\}_{n \in \mathbb{N}} \subset \mathcal{H}_{a}$ be a sequence converging to $g$ in the $\mathcal{H}_{K}$ norm. Since $\mathcal{H}_{K}$ is a RKHS, for each $z \in \mathbb{C}$ we have that $f_{n}(z) \rightarrow g(z)$; in particular $g(a)=\lim _{n \rightarrow \infty} f_{n}(a)=0$.

The following lemma relates de $\mathrm{ZR}_{a}$ property with the subspace $\mathcal{H}_{a}$ via the restriction of the operator $\mathfrak{S}_{0}^{*}$ to the subspace $\mathcal{H}_{0}$ :

Lemma 8. Assume that the operator $S_{0}$ is bounded. Let $\widetilde{\mathfrak{S}_{0}^{*}}$ be the restriction of the operator $\mathfrak{S}_{0}^{*}$ to the closed subspace $\mathcal{H}_{0}$ of $\mathcal{H}_{K}$. Given $a \in \mathbb{C}$, the $Z R_{a}$ property holds in $\mathcal{H}_{K}$ if and only if the range of the operator $I-a \widehat{\mathfrak{S}_{0}^{*}}$ is $\mathcal{H}_{a}$.

Proof. Assume that the operator $S_{0}$ is bounded and, therefore, the $\mathrm{ZR}_{0}$ property holds. The range of the operator $I_{0}-a \widetilde{\mathcal{S}_{0}^{*}}$ is a subspace included in $\mathcal{H}_{a}$, where $I_{0}:=I_{\mid \mathcal{H}_{0}}$. If the $\mathrm{ZR}_{a}$ property holds in $\mathcal{H}_{K}$ then any entire function $g$ in $\mathcal{H}_{a}$ can be written as $g(z)=z h(z)-a h(z)$ where $h \in \mathcal{H}_{K}$. The entire function $z h(z)$ belongs to $\mathcal{H}_{0}$ and $g=\left(I-a \widetilde{\mathfrak{S}_{0}^{*}}\right)(z h)$, i.e., $g$ belongs to the range of $I-a \widetilde{\mathfrak{S}_{0}^{*}}$.

Now, suppose that the range of $I-a \widetilde{\mathfrak{S}_{0}^{*}}$ is $\mathcal{H}_{a}$. For any $g \in \mathcal{H}_{a}$ there exists $f \in \mathcal{H}_{0}$ such that $g(z)=f(z)-a f(z) / z=(z-a) f(z) / z$. Hence, since the $\mathrm{ZR}_{0}$ property holds, the entire function $g(z) /(z-a)=f(z) / z$ belongs to $\mathcal{H}_{K}$.

In the sequel we follow the Fredholm operator theory as it appears in [7].

Theorem 9. Assume that the operator $S_{0}$ is bounded. Then, there exists $\delta>0$ such that the $Z R_{a}$ property holds in $\mathcal{H}_{K}$ for $|a|<\delta$.

Proof. The identity operator $I$ restricted to $\mathcal{H}_{0}$, i.e., $I_{0}$, is a Fredholm operator. Indeed, $I_{0}$ is bounded; its range is $R\left(I_{0}\right)=\mathcal{H}_{0}$, hence, closed; the kernel of $I_{0}$, $N\left(I_{0}\right)=\{0\}$ is finite dimensional and the codimension of the range is finite 
and equal to 1 (recall that $\mathcal{H}_{0}^{\perp}$ is the subspace of $\mathcal{H}_{K}$ generated by $f_{0}(z)=$ $\left.\left\langle K(z), c_{n}(0)\right\rangle, z \in \mathbb{C}\right)$. The index of $I_{0}$ is $\operatorname{dim} N\left(I_{0}\right)-\operatorname{codim} R\left(I_{0}\right)=-1$.

For any $a \in \mathbb{C}$ the operator $I_{0}-a \widetilde{\mathcal{S}_{0}^{*}}$ is injective. Indeed, let $f \in \mathcal{H}_{0}$ such that $\left(I_{0}-a \widetilde{\mathfrak{S}_{0}^{*}}\right) f=0$ or, equivalently, such that $\frac{z-a}{z} f(z)=0$, for any $z \in \mathbb{C}$. This implies that $f$ is the zero function since $f$ is an entire function. Following see $\left[7\right.$, p. 34], there exists $\delta>0$ such that if $|a|<\delta$ the operator $I_{0}-a \widetilde{\mathfrak{S}_{0}^{*}}$ is Fredholm and its index verifies ind $\left(I_{0}-a \widetilde{\mathfrak{S}_{0}^{*}}\right)=$ ind $I_{0}=-1$. Since $I_{0}-a \widetilde{\mathfrak{S}_{0}^{*}}$ is an injective Fredholm operator we have that $R\left(I_{0}-a \widetilde{\mathfrak{S}_{0}^{*}}\right)=\mathcal{H}_{a}$. Hence, by Lemma 8 the $\mathrm{ZR}_{a}$ property holds in $\mathcal{H}_{K}$

An estimation of the constant $\delta$ is given in next proposition:

Proposition 7. Assume that the operator $S_{0}$ is bounded. Then, the $Z R_{a}$ holds for each $a \in \mathbb{C}$ such that $|a|<\left\|\widetilde{\mathfrak{S}_{0}^{*}}\right\|^{-1}$.

Proof. The numerical range of the operator $\widetilde{\mathfrak{S}_{0}^{*}}$ is defined by:

$$
\left.\Theta\left(\widetilde{\mathfrak{S}_{0}^{*}}\right)=\left\{\widetilde{\left\langle\widetilde{\mathfrak{S}}_{0}^{*}\right.} f, f\right\rangle \mid f \in \mathcal{H}_{0} \text { and }\|f\|=1\right\} .
$$

Since $\widetilde{\mathfrak{S}_{0}^{*}}$ is bounded we have that $\Theta\left(\widetilde{\mathfrak{S}_{0}^{*}}\right)$ is bounded in $\mathbb{C}$; indeed, $\left|\widetilde{\mathfrak{S}_{0}^{*} F}, F\right\rangle \mid \leq\left\|\widetilde{\mathfrak{S}_{0}^{*}}\right\|$.

It is known that if $|\lambda|>\left\|\widetilde{\mathfrak{S}_{0}^{*}}\right\|$ then $\lambda I_{0}-\widetilde{\mathfrak{S}_{0}^{*}}$ is an injective semi-Fredholm operator, whose range, $R\left(\lambda I_{0}-\widetilde{\mathfrak{S}_{0}^{*}}\right)$, is closed and codim $R\left(\lambda I_{0}-\widetilde{\mathfrak{S}_{0}^{*}}\right)$ is constant in the set $\left\{\mu \in \mathbb{C}\right.$ such that $\left.|\mu|>\left\|\widetilde{\widetilde{S}_{0}^{*}}\right\|\right\}$ (see $[7$, p. 100]).

Let $\lambda=a^{-1}$, taking into account that $a^{-1} I_{0}-\widetilde{\mathcal{S}_{0}^{*}}: \mathcal{H}_{0} \rightarrow \mathcal{H}_{a}$ we obtain that if $|a|<\left\|\widetilde{\mathfrak{S}_{0}^{*}}\right\|^{-1}$ then $R\left(a^{-1} I_{0}-\widetilde{\mathfrak{S}_{0}^{*}}\right)$ is a closed subspace in $\mathcal{H}_{a}$, therefore, $\operatorname{codim} R\left(a^{-1} I_{0}-\widetilde{\mathfrak{S}_{0}^{*}}\right)=C$ with $C \geq 1$ for each $a \neq 0$ satisfying $|a|<\left\|\widetilde{\mathfrak{S}_{0}^{*}}\right\|^{-1}$. From Theorem 9 we have that if $b \neq 0$ is close to 0 then $\operatorname{codim} R\left(I_{0}-b \widetilde{\mathfrak{S}_{0}^{*}}\right)=\operatorname{codim} R\left(b^{-1} I_{0}-\widetilde{\mathfrak{S}_{0}^{*}}\right)=1$. Hence, $C=1$ and the $\mathrm{ZR}_{a}$ property holds in $\mathcal{H}_{K}$ whenever $|a|<\left\|\widetilde{\mathfrak{S}_{0}^{*}}\right\|^{-1}$.

Corollary 10. Assume that the mapping $\mathcal{T}_{K}$ in (4) is injective, the sequence $\left\{c_{n}(a)\right\}_{n=1}^{\infty}$ is linearly independent for any $a \in \mathbb{C}$ and $1 \in \mathcal{H}_{K}$. Then the set

$$
\left\{b \in \mathbb{C} \mid \text { property } Z R_{b} \text { holds }\right\}
$$

is an open set in $\mathbb{C}$

Proof. It is a straightforward consequence of Theorems 5 and 9.

Remark As far as Theorem 9 is concerned, one can construct kernels $K$ such that the $\mathrm{ZR}_{a}$ property at a fixed point $a \in \mathbb{C}$ implies that the zero-removing property holds in $\mathcal{H}_{K}$ for every $b \in \mathbb{C}$. It remains the open question whether this is true for every space $\mathcal{H}_{K}$. 


\section{The Differentiation Operator in $\mathcal{H}_{K}$}

In general, the differentiation operator $\mathcal{D}: \mathcal{H}_{K} \longrightarrow \mathcal{H}_{K}$ given by $\mathcal{D}(f)=$ $f^{\prime}, f \in \mathcal{H}_{K}$, is not well-defined as the following example shows. In example (e) in Sect. 2 with $\gamma:=\{\sqrt{n !}\}_{n \in \mathbb{N}_{0}}$, an entire function $f(z)=\sum_{n=0}^{\infty} \alpha_{n} z^{n}$ belongs to $\mathcal{H}_{K_{\gamma}}$ if and only if the sequence $\left\{\sqrt{n !} \alpha_{n}\right\}_{n=0}^{\infty}$ belongs to $\ell^{2}\left(\mathbb{N}_{0}\right)$. In particular, for the sequence $\alpha_{n}=1 /(n \sqrt{n !}), n \in \mathbb{N}_{0}$, the corresponding function $f$ belongs to $\mathcal{H}_{K_{\gamma}}$; however its derivative $f^{\prime}$ does not belong to $\mathcal{H}_{K_{\gamma}}$. A sufficient condition is given in the next result:

Theorem 11. Suppose that the sequence $\left\{c_{n}(0)\right\}_{n \in \mathbb{N}_{0}}$ of Taylor coefficients of $K$ at 0 is complete and minimal in $\mathcal{H}$. Consider the numbers $\delta_{n}>0, n \in \mathbb{N}_{0}$, given in (13). If the series

$$
\sum_{n=0}^{\infty} \frac{(n+1)}{\delta_{n}} \frac{\left\|c_{n+1}(0)\right\|}{\left\|c_{n}(0)\right\|}
$$

converges, then the differentiation operator $\mathcal{D}$ is a well-defined bounded operator on $\mathcal{H}_{K}$.

Proof. Let $f$ be in $\mathcal{H}_{K}$; there exists $x \in \mathcal{H}$ such that $f(z)=\langle K(z), x\rangle$, for any $z \in \mathbb{C}$, and $f(z)=\sum_{n=0}^{\infty}\left\langle c_{n}(0), x\right\rangle z^{n}$. Therefore,

$$
f^{\prime}(z)=\sum_{n=1}^{\infty}\left\langle c_{n}(0), x\right\rangle n z^{n-1}, \quad z \in \mathbb{C} .
$$

The derivative $f^{\prime}$ of the entire function $f$ belongs to $\mathcal{H}_{K}$ if and only if there exists $y \in \mathcal{H}$ such that

$$
\left\langle c_{n}(0), y\right\rangle=(n+1)\left\langle c_{n+1}(0), x\right\rangle \quad \text { for any } n \in \mathbb{N}_{0} .
$$

Proceeding as in the proof of Theorem 4, the set of equations (18) has a solution $y \in \mathcal{H}$ if and only if the operator

$$
\begin{aligned}
\mathfrak{D}: \operatorname{span}\left\{c_{n}(0)\right\}_{n \in \mathbb{N}_{0}} & \longrightarrow \operatorname{span}\left\{c_{n}(0)\right\}_{n \in \mathbb{N}_{0}} \\
c_{n}(0) & \longmapsto(n+1) c_{n+1}(0),
\end{aligned}
$$

is bounded. Let $u=\sum_{n} a_{n} c_{n}(0)$ be a finite sum in $\mathcal{H}$ with $\|u\|_{\mathcal{H}}=1$. By using inequalities in (14), we obtain

$$
\begin{aligned}
\|\mathfrak{D} u\|= & \left\|\sum_{n} a_{n}(n+1) c_{n+1}(0)\right\| \leq \sum_{n}(n+1)\left|a_{n}\right|\left\|c_{n+1}(0)\right\| \\
& \leq \sum_{n} \frac{(n+1)}{\delta_{n}} \frac{\left\|c_{n+1}(0)\right\|}{\left\|c_{n}(0)\right\|} .
\end{aligned}
$$

Hence, the convergence of the series in (17) implies the continuity of the operator $\mathfrak{D}$. Moreover, the boundedness of the operator $\mathfrak{D}$ implies the boundedness of the differentiation operator $\mathcal{D}$. Indeed, if $\mathfrak{D}$ is bounded on $\operatorname{span}\left\{c_{n}(0)\right\}_{n=0}^{\infty}$ then it can be extended by continuity to the whole space $\mathcal{H}$. 
In this case, the adjoint operator of $\mathfrak{D}, \mathfrak{D}^{*}: \mathcal{H} \rightarrow \mathcal{H}$ is bounded and it is straightforward to prove that $\mathcal{D}=\mathcal{T}_{K} \mathfrak{D}^{*} \mathcal{T}_{K}^{-1}$ where $\mathcal{T}_{K}: \mathcal{H} \rightarrow \mathcal{H}_{K}$ is the anti-linear isometry defined in (4).

Moreover, whenever the differentiation operator $\mathcal{D}$ is a well-defined bounded operator on $\mathcal{H}_{K}$, the translation operator given by $T_{a} f(z):=f(z-a)$, $z \in \mathbb{C}$, is also a well-defined bounded operator $T_{a}: \mathcal{H}_{K} \longrightarrow \mathcal{H}_{K}$ for each $a \in \mathbb{C}$. Indeed, adapting a result from [5] we obtain:

Proposition 8. Suppose that the differentiation operator $\mathcal{D}$ defined as $\mathcal{D}(f)=f^{\prime}$ is a well-defined bounded operator $\mathcal{D}: \mathcal{H}_{K} \rightarrow \mathcal{H}_{K}$. Then, for each a $\in \mathbb{C}$, the translation operator $T_{a}: \mathcal{H}_{K} \rightarrow \mathcal{H}_{K}$ is a well-defined, bounded operator. Moreover, we have the following expansion for $T_{a}$ converging in the operator norm

$$
T_{a}=\sum_{n=0}^{\infty} \frac{(-a)^{n}}{n !} \mathcal{D}^{n}
$$

Proof. It is a well-known result that (19) holds in $\mathcal{E}$, the space of entire functions endowed with the topology of the uniform convergence on compact sets (see, for instance, [5]). Since the differentiation operator $\mathcal{D}$ is bounded on the Hilbert space $\mathcal{H}_{K}$, the series on the right side of (19) converges absolutely, and hence in the operator norm to a bounded operator on $\mathcal{H}_{K}$. As the convergence in $\mathcal{H}_{K}$ implies convergence in the space $\mathcal{E}$, this operator must be $T_{a}$.

Note that, under the hypotheses of Theorem 11, in the corresponding $\mathcal{H}_{K}$ space the ZR property holds. Indeed, by using Lemma $(6)$ ) the $Z_{R_{0}}$ property holds. For $a \in \mathbb{C} \backslash\{0\}$, let $g$ be an entire function in $\mathcal{H}_{K}$ such that $g(a)=0$. The entire function $f=T_{-a} g$ belongs to $\mathcal{H}_{K}$ and $f(0)=g(a)=0$. Since the $\mathrm{ZR}_{0}$ property holds we have

$$
h(z)=\frac{f(z)}{z}=\frac{g(z+a)}{z} \in \mathcal{H}_{K},
$$

and hence $g(z) /(z-a)=\left(T_{a} h\right)(z) \in \mathcal{H}_{K}$.

Closing the paper, it is worth to mention that the convergence of the series in (17) imposes a condition on the rate of decay of the sequence $\left\{\left\|c_{n}(0)\right\|\right\}_{n \in \mathbb{N}_{0}}$ and therefore, on the growth of the functions in $\mathcal{H}_{K}$. Indeed, let $F$ be the entire function defined by $F(z)=\sum_{n=0}^{\infty}\left\|c_{n}(0)\right\| z^{n}$. Then, for any $f \in \mathcal{H}_{K}$, we have

$$
|f(z)| \leq\|f\| F(|z|)=\|f\| \sum_{n=0}^{\infty}\left\|c_{n}(0)\right\||z|^{n} \quad \text { for all } z \in \mathbb{C} .
$$

In order to illustrate the relationship between the decaying of the sequence $\left\{\left\|c_{n}(0)\right\|\right\}_{n \in \mathbb{N}_{0}}$, the growth of functions in $\mathcal{H}_{K}$ and the ZR property, suppose that

$$
\lim _{n \rightarrow \infty} n^{r} \frac{\left\|c_{n}(0)\right\|}{\left\|c_{n-1}(0)\right\|}=\alpha \quad \text { for some } r>2
$$


Assume that the sequence $\left\{c_{n}(0)\right\}_{n \in \mathbb{N}_{0}}$ is uniformly minimal, i.e., there exists $\delta$ such that $\delta_{n}>\delta>0$ for any $n \in \mathbb{N}_{0}$ (see [12, p. 27]). Notice that the existence of the limit in (22) implies the convergence of the series $\sum_{n=0}^{\infty}(n+$ 1) $\frac{\left\|c_{n+1}(0)\right\|}{\left\|c_{n}(0)\right\|}$ and, the boundedness of the differentiation operator on $\mathcal{H}_{K}$; as a consequence, the ZR property holds.

Let $\gamma>\alpha$; following [5], condition (22) implies the existence of a positive constant $C$, depending only on $\gamma$, such that

$$
\left\|c_{n}(0)\right\| \leq C\left(\frac{\mathrm{e} \gamma^{1 / r}}{n}\right)^{n r}, \quad n \in \mathbb{N}_{0} .
$$

Now according to $[17$, p. 7$]$, the entire function $g$ defined by

$$
g(z)=\sum_{n=1}^{\infty}\left(\frac{\mathrm{e} M r^{-1}}{n}\right)^{n r} z^{n}
$$

where $M=r \gamma^{1 / r}$, has order $r^{-1}$. Having in mind (23) we obtain that $F(|z|) \leq$ $C g(|z|)$ for any $z \in \mathbb{C}$. Hence, inequality (21) implies that any function $f$ in $\mathcal{H}_{K}$ has order less or equal than $r^{-1}<1 / 2$.

\section{Acknowledgements}

The authors are indebted to the anonymous referees for their valuable suggestions. This work has been supported by the grant MTM2009-08345 from the Spanish Ministerio de Ciencia e Innovación (MICINN).

\section{References}

[1] Baranov, A.D.: Polynomials in the de Branges spaces of entire functions. Ark. Mat. 44(1), 16-38 (2006)

[2] Belov, Y., Mengestie, T.Y., Seip, K.: Unitary discrete Hilbert transforms. J. Anal. Math. 112, 383-393 (2010)

[3] Belov, Y., Mengestie, T.Y., Seip, K.: Discrete Hilbert transforms on sparse sequences. Proc. Lond. Math. Soc. 103(1), 73-105 (2011)

[4] de Branges, L.: Hilbert Spaces of Entire Functions. Prentice-Hall, Englewood Cliffs (1968)

[5] Chan, K.C., Shapiro, J.: The cyclic behavior of traslation operators on Hilbert space of entire functions. Indiana Univ. Math. J. 40(4), 1421-1449 (1991)

[6] Christensen, O.: An Introduction to Frames and Riesz Bases. Birkhäuser, Boston (2003)

[7] Edmunds, D.E., Evans, W.D.: Spectral Theory and Differential Operators. Oxford Science, Oxford (1990)

[8] Everitt, W.N., García, A.G., Hernández-Medina, M.A.: On Lagrange-type interpolation series and analytic Kramer kernels. Results Math. 51, 215-228 (2008) 
[9] Fernández-Moncada, P.E., García, A.G., Hernández-Medina, M.A.: The zeroremoving property and Lagrange-type interpolation series. Numer. Funct. Anal. Optim. 32, 858-876 (2011)

[10] García, A.G., Hernández-Medina, M.A., Szafraniec, F.H.: Analytic Kramer kernels, Lagrange-type interpolation series and de Branges spaces. Complex Var. Elliptic Equ. 58(1), 79-97 (2013)

[11] Godefroy, G., Shapiro, J.: Operators with dense, invariant cyclic vector manifolds. J. Funct. Anal. 98, 229-269 (1991)

[12] Gurariy, V.I., Lusky, W.: Geometry of Müntz spaces and related questions. Springer, New York (2005)

[13] Han, D., Nashed, M.Z., Sun, Q.: Sampling expansions in reproducing kernel Hilbert and Banach spaces. Numer. Funct. Anal. Optim. 30, 971-987 (2009)

[14] Havin, V.P., Mashreghi, J.: Admissible majorants for model subspace of $H^{2}$. Part I: slow winding of the generating inner function. Can. J. Math. 55, 1231$1263(2003)$

[15] Havin, V.P., Mashreghi, J.: Admissible majorants for model subspace of $H^{2}$. Part II: Fast winding of the generating inner function. Can. J. Math. 55, 12641301 (2003)

[16] Kramer, H.P.: A generalized sampling theorem. J. Math. Phys. 63, 68-72 (1959)

[17] Levin B.Y.: Lectures on Entire Functions. Translations of Mathematical Monographs, vol. 150. AMS, Providence (1996)

[18] Mee, C.van der, Nashed, M.Z., Seatzu, S.: Sampling expansions and interpolation in unitarily translation invariant reproducing kernel Hilbert spaces. Adv. Comput. Math. 19, 355-372 (2003)

[19] Nashed, M.Z., Walter, G.G.: General sampling theorems in reproducing kernel Hilbert spaces. Math. Control Signals Syst. 4, 373-412 (1991)

[20] Nashed, M.Z., Sun, Q.: Sampling and reconstruction of signals in a reproducing kernel subspace of $L^{p}\left(\mathbb{R}^{d}\right)$. J. Funct. Anal. 258, 2422-2452 (2010)

[21] Nashed M.Z., Sun Q.: Function spaces for sampling expansions. In: Xhen, X., Zayed, A. (eds.) Multiscale Signal Analysis and Modeling, pp. 81-104. Lecture Notes in Electrical Engineering. Springer, New York (2012)

[22] Nikolskii, N.K.: Treatise on Shift Operator. Springer, New York (1986)

[23] Partington, J.R.: Interpolation, Identification and Sampling. Clarendon Press, Oxford (1997)

[24] Saitoh, S.: Integral transforms, reproducing kernels and their applications. Longman, Essex (1997)

[25] Seip, K.: Interpolation and Sampling in Spaces of Analytic Functions. University Lecture Series, vol. 33. AMS, Providence (2004)

[26] Taylor, A.E., Lay, D.C.: Introduction to Functional Analysis. Wiley, New York (1980)

[27] Woracek, H.: De Branges spaces of entire functions closed under forming difference quotients space of entire functions. Integral Equ. Oper. Theory 37(2), 238249 (2000) 
[28] Young, R.M.: An Introduction to Nonharmonic Fourier Series. Academic Press, New York (2001)

A. G. García

Departamento de Matemáticas

Universidad Carlos III de Madrid

Avda. de la Universidad 30

28911 Leganés-Madrid, Spain

e-mail: agarcia@math.uc3m.es

M. A. Hernández-Medina

Departamento de Matemática Aplicada

E.T.S.I.T., U.P.M.

Avda. Complutense 30, 28040 Madrid, Spain

e-mail: miguelangel hernandez.medina@upm .es

Received: September 18, 2013

Accepted: September 4, 2014. 